\title{
Erratum to: volume 73, issue 3 of Journal of Physiology and Biochemistry
}

\section{The Publisher}

Published online: 5 October 2017

(C) University of Navarra 2017

From the title : J Physiol Biochem Volume 73, issue 3

https://doi.org/10.1007/s13105-017-0567-z
https://doi.org/10.1007/s13105-017-0555-3
https://doi.org/10.1007/s13105-017-0557-1
https://doi.org/10.1007/s13105-017-0558-0
https://doi.org/10.1007/s13105-017-0559-z
https://doi.org/10.1007/s13105-017-0561-5
https://doi.org/10.1007/s13105-017-0563-3
https://doi.org/10.1007/s13105-017-0565-1
https://doi.org/10.1007/s13105-017-0566-0
https://doi.org/10.1007/s13105-017-0568-y

The online version of the original articles can be found at

https://doi.org/10.1007/s13105-017-0567-z

https://doi.org/10.1007/s13105-017-0555-3 https://doi.org/10.1007/s13105-017-0557-1 https://doi.org/10.1007/s13105-017-0558-0 https://doi.org/10.1007/s13105-017-0559-Z https://doi.org/10.1007/s13105-017-0561-5 https://doi.org/10.1007/s13105-017-0563-3 https://doi.org/10.1007/s13105-017-0565-1 https://doi.org/10.1007/s13105-017-0566-0 https://doi.org/10.1007/s13105-017-0568-y https://doi.org/10.1007/s13105-017-0572-2 https://doi.org/10.1007/s13105-016-0544-y https://doi.org/10.1007/s13105-016-0547-8 https://doi.org/10.1007/s13105-017-0552-6 https://doi.org/10.1007/s13105-017-0556-2 https://doi.org/10.1007/s13105-017-0560-6 https://doi.org/10.1007/s13105-016-0526-0

The Publisher

Springer Science+Business Media B.V., The Netherlands

\begin{abstract}
https://doi.org/10.1007/s13105-017-0572-2
https://doi.org/10.1007/s13105-016-0544-y

https://doi.org/10.1007/s13105-016-0547-8

https://doi.org/10.1007/s13105-017-0552-6

https://doi.org/10.1007/s13105-017-0556-2

https://doi.org/10.1007/s13105-017-0560-6

https://doi.org/10.1007/s13105-016-0526-0
\end{abstract}

Volume 73 issue 3 was published with an incorrect cover date. Correct is August 2017. The Publisher apologizes for this mistake and all related inconveniences caused by this. 